\title{
Enhancing the quality of life for palliative care cancer patients in Indonesia through family caregivers: a pilot study of basic skills training
}

\author{
Martina Sinta Kristanti ${ }^{*}$ Sri Setiyarini and Christantie Effendy
}

\begin{abstract}
Background: Palliative care in Indonesia is problematic because of cultural and socio-economic factors. Family in Indonesia is an integral part of caregiving process in inpatient and outpatient settings. However, most families are not adequately prepared to deliver basic care for their sick family member. This research is a pilot project aiming to evaluate how basic skills training (BST) given to family caregivers could enhance the quality of life (QoL) of palliative care cancer patients in Indonesia.

Methods: The study is a prospective quantitative with pre and post-test design. Thirty family caregivers of cancer patients were trained in basic skills including showering, washing hair, assisting for fecal and urinary elimination and oral care, as well as feeding at bedside. Patients' QoL were measured at baseline and 4 weeks after training using EORTC QLQ C30. Hypothesis testing was done using related samples Wilcoxon Signed Rank. A paired t-test and one-way ANOVA were used to check in which subgroups was the intervention more significant.

Results: The intervention showed a significant change in patients' global health status/QoL, emotional and social functioning, pain, fatigue, dyspnea, insomnia, appetite loss, constipation and financial hardship of the patients. Male patient's had a significant effect on global health status (qol) $(p=0.030)$; female patients had a significant effect on dyspnea $(p=0.050)$ and constipation ( $p=0.038)$. Younger patients had a significant effect in global health status/ QoL $(p=0.002)$. Patients between 45 and 54 years old had significant effect on financial issue $(p=0.039)$. Caregivers between 45 and 54 years old had significant effect on patients' dyspnea $(p=0.031)$.

Conclusions: Basic skills training for family caregivers provided some changes in some aspects of QoL of palliative cancer patients. The intervention showed promises in maintaining the QoL of cancer patients considering socioeconomic and cultural challenges in the provision of palliative care in Indonesia.
\end{abstract}

Keywords: Indonesia, Basic skills training, Palliative care, Quality of life, Cancer

\section{Background}

Chronic non-communicable diseases such as cancer pose a major, ongoing public health problem and are responsible for $60 \%$ of deaths in Southeast Asia [1]. These conditions are also strongly correlated with poverty. Surveillance of these diseases and their risk factors needs to be improved, and health care systems must be strengthened to address

\footnotetext{
*Correspondence: sinta@ugm.ac.id

School of Nursing, Faculty of Medicine, Universitas Gadjah Mada, Yogyakarta, Indonesia

(c) The Author(s). 2017 Open Access This article is distributed under the terms of the Creative Commons Attribution 4.0 International License (http://creativecommons.org/licenses/by/4.0/), which permits unrestricted use, distribution, and reproduction in any medium, provided you give appropriate credit to the original author(s) and the source, provide a link to the Creative Commons license, and indicate if changes were made. The Creative Commons Public Domain Dedication waiver (http://creativecommons.org/publicdomain/zero/1.0/) applies to the data made available in this article, unless otherwise stated.

the needs of the patients through primary health care and appropriate referral systems [1].

Similar to other Southeast Asian countries, Indonesia faces a range of challenges in providing health services for the poor. These include unavailability and unaffordability of services, as well as lack of adequate staff, transportation, and equipment [2]. Many patients in Indonesia tend to postpone cancer treatment because of their lack of knowledge on treatment options, insufficient financial resources, the side effects of treatment, and the paternalistic approach of health professionals 
[3]. The survival rate for cancer patients in Indonesia is resultantly lower compared with that of other Southeast Asian countries $[4,5]$.

Although a universal health insurance system has been in place in the country since 2005, it has not worked as well as planned [6, 7], and economically disadvantaged patients still typically have to rely on family members to care for them rather than seeking professional treatment [8]. Unsurprisingly, wealthier patients generally tend to have better treatment outcomes than financially disadvantaged ones [9].

In Indonesia, strong family bonds underpin the high degree of familial involvement during patients' care and hospitalization [10]. Nurses assume important roles in activities pertaining to daily living, physical, spiritual, social, psychosocial, autonomous, and financial aspects during periods of hospitalization. It has also been reported that physicians primarily focus on physical symptoms, and that supporting individuals such as social workers and volunteers play a largely insubstantial role [10]. At home, the family provides the majority of care, mostly with no expert support from visiting nurses, physiotherapists, or occupational therapists.

Indonesia still has a lack of formal institutions to support patients with long-term conditions, as seen in the absence of hospices and respite care. Therefore, disadvantaged patients with terminal illness are typically cared for at home by family members [8] who generally have little or no training in provision of basic care. The lack of necessary knowledge and skills may cause family caregivers to have a lack confidence and feelings of uncertainty, which can lead to unease and anxiety [11]. Family caregivers of patients with advanced stage cancer may have similar experiences [12]. Basic skills training (BST) for family caregivers is therefore crucial.

\section{Aim of study}

The research question underlying the present pilot study pertained to the effects that BST for family caregivers had on cancer patients' quality of life (QoL). Using twotailed testing, an intervention was tested to determine how BST affect the QoL of palliative cancer patients.

\section{Methods}

\section{Participants}

Head nurses in selected cancer wards in the major teaching hospital in Yogyakarta identified patients who could potentially serve as research participants. Inclusion criteria were: (i) stage 3 or 4 cancer, (ii) Palliative Performance Scale (PPS) score of less than 60, (iii) patients and family caregivers consented to participating in the study, and (iv) family caregivers were functionally literate. Following an examination of medical charts, 41 potential participants and their family caregivers were found to meet the study inclusion criteria and agreed to participate. Of these, 30 continued for the duration of the study.

\section{Outcome measurement}

The major outcome variable, QoL, was assessed using the European Organization for Research and Treatment Cancer Quality of Life C30 version 3 (EORTC QOQL C30) $[13,14]$. This instrument is used worldwide and has been translated into more than 60 languages, including Indonesian [15]. The EORTC QLQ-30 consists of 30 questions divided into three subscales: global health status/QoL, functional scales, and symptom/single items. There are only two questions on global health status/QoL scale. The functional scale consists of five subscales: physical (PF), role (RF), emotional (EF), cognitive (CF), and social (SF). Symptom/single items consist of nine discrete variables: fatigue (FA), nausea/vomiting (NV), pain (PA), dyspnea (DY), insomnia/sleep (SL), appetite loss (AP), constipation (CO), diarrhea (DI), and financial hardship related to illness (FI) [13].

The functional and symptom/single subscales are measured on a four-point Likert scale, from 1 ("not at all") to 4 ("very much"). An exception is for the global health status/QoL scale, which is based on a seven-point Likert scale, from 1 ("very poor") to 7 ("excellent") [13]. For Functional and Global health status/QoL, a higher score indicates a better QoL. In contrast, a higher score for symptoms and single items indicates poorer QoL [14].

\section{Intervention}

We planned an intervention centered on a BST educational package that we developed to encourage interaction between family caregivers and nurse educators $[11,16]$. The package included a 1-h video (on CD) and a module consisting of five chapters on assisting a bedridden patient with bathing, providing oral hygiene, hair washing, assisting with urination and bowel movements, and managing feeding orally and using a nasogastric tube. Nurse educators demonstrated these skills and provided assistance sessions to family caregivers for practicing their skills on patients. The nurse educators were given a 1-day training in advance about the research objectives and procedures, measurement of research outcomes, and patient education theory.

\section{Data collection}

Three training sessions for the family caregivers were provided (Fig. 1). The first was held at the hospital 1 week prior to the patient's discharge. The family caregivers observed an initial demonstration by the nurse educators, watched the video, and then practiced the skills. Prior to this, the selected patients had provided signed informed consent, and baseline data on the patients' QoL were collected. 


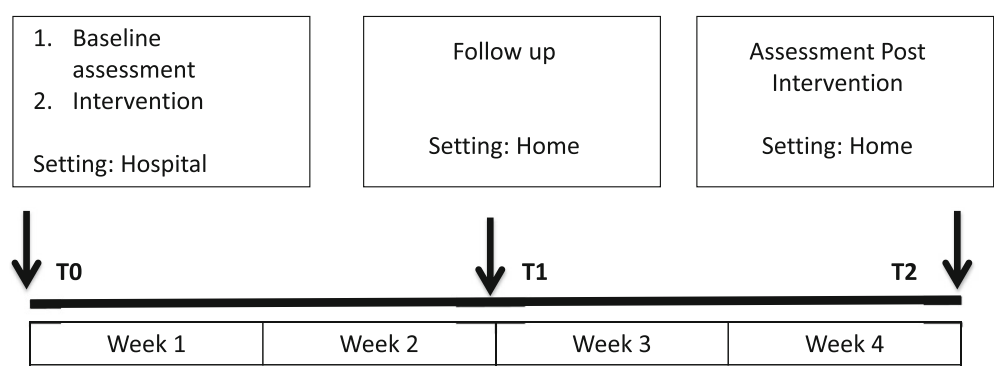

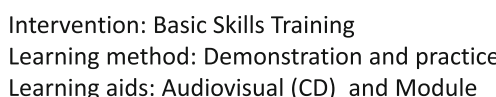

Learning aids: Audiovisual (CD) and Module

Outcome measurement: EORTC QLQ C30

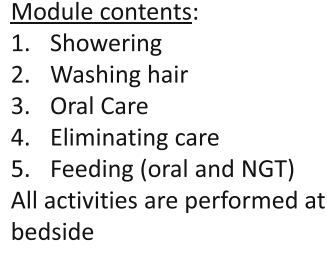

Fig. 1 Time line and intervention details

Two weeks later, the nurse educators visited individual patients' homes to give encouragement to the family caregivers and assist them in developing their skills in providing personal care, and to provide further information if required. On the third visit, within 1 month after patients' discharge from the hospital, nurse educators visited the patients' homes and collected further data on the patients' QoL. An interval of 2 weeks after the second visit was chosen as the most suitable time between the follow up visits because it was reported in a similar study that $39.3 \%$ of cancer patients in a palliative condition passed away within 3 weeks [17].

\section{Analysis}

Data were cleaned and assessed for normality, descriptive analysis was performed using frequency and mean to analyze patient demographics and QoL. The paired data testing was conducted using related samples with Wilcoxon Signed Rank tes. Cohen's $d$ test was used to calculate the effect size; scores of $0.2,0.3$, and 0.8 were considered small, moderate, and high, respectively [16]. A paired t-test and one-way analysis of variance (ANOVA) were used to investigate in which subgroups an intervention might be effective. Statistical significance was set at $p<0.05$.

\section{Results}

Of the 41 participants who entered the study, one decided not to continue, three could not be reached for contact after the second visit, and seven died before the program was evaluated. This left 30 participants who completed the study; their results are reported herein (Table 1).

\section{Characteristic of participants}

As shown in Table 1, the ratio of female to male participants was around 3:1. Most patients were above 55 years old (37\%) and approximately one-third were diagnosed with breast cancer. Most participants were not newly diagnosed, as they reported having been informed more than 5 months prior. Over $60 \%$ of the participants had a monthly income of less than USD 100. Ten of 30 received chemotherapy only and most received neither chemotherapy nor radiotherapy (47\%). A closer inspection revealed that $77 \%$ had moderate functional ability based on their PPS score, while $23 \%$ had poor functional ability. Fifty percent of participants were being cared for by their spouses and $60 \%$ of the family caregivers were female. Sixty percent of family caregivers were under 44 years old, $80 \%$ lived with the patients, over half had graduated from senior high school, and $60 \%$ had no previous caregiving experience.

\section{Quality of life}

In general, scores for almost all items in the EORTC QLQ C30 increased after intervention. As shown in Table 2, participants' global health status/QoL ratings significantly improved after intervention from $M=40.27$; $\mathrm{SD}=17.79$ to $M=56.94 ; \mathrm{SD}=18.05$ with $p=0.001$. For the functional scales, EF and SF improved after intervention, with some positive changes in $\mathrm{PF}, \mathrm{CF}$, and RF. Those last three items did not improve significantly after intervention ( $p=0.225,0.418$, and 0.431 , respectively). As for symptoms and single items, there were significant reductions in FA $(p=0.022)$, PA $(p=0.028)$, DY $(p=0.02)$, $\mathrm{SL}(p=0.013), \mathrm{AP}(p=0.030), \mathrm{CO}(p=0.004)$, and FI $(p=$ $0.009)$. The other symptoms, such as nausea and diarrhea, showed declining trends, but were not statistically significant ( $p=0.243$ and 0.097 , respectively). The majority of 
Table 1 Demographics data

\begin{tabular}{|c|c|c|}
\hline Characteristics & $\begin{array}{l}\text { Patient } \\
(n=30)\end{array}$ & $\begin{array}{l}\text { Caregiver } \\
(n=30)\end{array}$ \\
\hline \multicolumn{3}{|l|}{ Sex, $n(\%)$} \\
\hline Male & $8(27)$ & $12(40)$ \\
\hline Female & $22(73)$ & $18(60)$ \\
\hline \multicolumn{3}{|l|}{ Age in years } \\
\hline $18-44$ & $9(30)$ & $18(60)$ \\
\hline $45-54$ & $10(33)$ & $8(27)$ \\
\hline$>55$ & $11(37)$ & $4(13)$ \\
\hline \multicolumn{3}{|l|}{ Type of Cancer } \\
\hline Breast & $9(30)$ & \\
\hline Digestive (colon, recti, sigmoid) & $5(17)$ & \\
\hline Gynaecology (Vulva, Ovarian, Cervical) & $5(17)$ & \\
\hline Non Hodgkin Lymphoma & $4(13)$ & \\
\hline Head and Neck & $4(13)$ & \\
\hline Osteosarcoma & $2(7)$ & \\
\hline Thyroid & $1(3)$ & \\
\hline \multicolumn{3}{|l|}{ Time since diagnoses (in months) } \\
\hline 1 to 3 & $7(23)$ & \\
\hline 3 to 5 & $5(17)$ & \\
\hline$>5$ & $18(60)$ & \\
\hline \multicolumn{3}{|l|}{ Income } \\
\hline$<100$ USD/month & $19(63)$ & \\
\hline $100-300$ USD/month & $8(27)$ & \\
\hline > 300 USD/month & $3(10)$ & \\
\hline \multicolumn{3}{|l|}{ Palliative Performance Score (PPS) } \\
\hline$<30$ (Poor functioning) & $7(23)$ & \\
\hline 40 - 60 (Moderate functioning) & $23(77)$ & \\
\hline \multicolumn{3}{|l|}{ Treatment } \\
\hline Chemotherapy only & $10(33)$ & \\
\hline Radiotherapy only & $1(3)$ & 3 \\
\hline Both chemotherapy \& radiotherapy & $5(17)$ & 17 \\
\hline None & $14(47)$ & 47 \\
\hline \multicolumn{3}{|l|}{ Education background } \\
\hline Elementary school & $9(30)$ & $5(17)$ \\
\hline Junior high school & $6(20)$ & $4(13)$ \\
\hline Senior high school & $11(37)$ & $16(53)$ \\
\hline University & $4(13)$ & $5(17)$ \\
\hline \multicolumn{3}{|l|}{ Relationship with patient } \\
\hline Spouse & & $15(50)$ \\
\hline Non-spouse & & $15(50)$ \\
\hline \multicolumn{3}{|l|}{ Live with patient } \\
\hline Yes & & $24(80)$ \\
\hline No & & $6(20)$ \\
\hline
\end{tabular}

Table 1 Demographics data (Continued)

\begin{tabular}{ll}
\hline Status of employment & \\
Work & $15(50)$ \\
Unemployed & $15(50)$ \\
Experience in caregiving & \\
Yes & $12(40)$ \\
No & $18(60)$
\end{tabular}

effect sizes were small to medium, at $0.10-0.53$, except for global health status/QoL, at 0.92 .

Table 3 shows that some subgroups had a significant effect on QoL. There was a significant difference related to the patient's sex on global health status/QoL $(p=0.038)$, DY $(p=0.046)$, and CO $(p=0.030)$. The patient's age significantly affected the scales of global health status/QoL $(p=0.002)$ and FI $(p=0.039)$. There were also significant effects of the caregivers' age on DY $(p=0.031)$ and caregivers' experience on DY $(p=0.030)$.

\section{Discussion}

The aim of the current study is piloting and testing an intervention given to the family caregivers on the quality of life of palliative cancer patients. As indicated in the Results section, there were several items on the QoL

Table 2 QOL score (EORTC QLQ C-30) pre and post intervention $(n=30)$

\begin{tabular}{|c|c|c|c|c|}
\hline \multirow[t]{2}{*}{ Subscale } & \multicolumn{2}{|l|}{ Mean score } & \multirow[t]{2}{*}{ Effect size } & \multirow[t]{2}{*}{$P$-value } \\
\hline & Pre (SD) & Post (SD) & & \\
\hline $\begin{array}{l}\text { Global health } \\
\text { status/QOL }^{\mathrm{a}}\end{array}$ & $40.27(17.79)$ & $56.94(18.05)$ & 0.92 & 0.001 \\
\hline \multicolumn{5}{|l|}{ Functional Scales ${ }^{b}$} \\
\hline Physical (PF) & $11.98(15.98)$ & $17.11(22 / 8)$ & 0.26 & 0.225 \\
\hline Role (RF) & $11.11(19.24)$ & $13.33(21.62)$ & 0.10 & 0.418 \\
\hline Emotional (EF) & $63.33(30.21)$ & $79.44(26.77)$ & 0.53 & 0.003 \\
\hline Cognitive (CF) & $73.89(24.24)$ & $75.55(27.24)$ & 0.06 & 0.431 \\
\hline Social (SF) & $20.56(25.40)$ & $35.56(33.82)$ & 0.50 & 0.012 \\
\hline \multicolumn{5}{|c|}{ Symptoms/Single Items ${ }^{\mathrm{b}}$} \\
\hline Fatigue (FA) & $68.33(24.20)$ & $56.29(28.12)$ & 0.45 & 0.022 \\
\hline Nausea (NV) & $25.00(28.28)$ & $20.55(24.24)$ & 0.16 & 0.243 \\
\hline Pain (PA) & $72.22(33.99)$ & $57.22(34.35)$ & 0.43 & 0.028 \\
\hline Dyspnoea (DY) & $38.89(39.22)$ & $12.22(28.34)$ & 0.77 & 0.002 \\
\hline Insomnia (SL) & $57.78(66.67)$ & $35.56(36.04)$ & 0.41 & 0.013 \\
\hline Appetite Loss (AP) & $60.00(39.53)$ & $44.44(36.40)$ & 0.40 & 0.030 \\
\hline Constipation (CO) & $32.22(38.63)$ & $20.00(34.57)$ & 0.33 & 0.004 \\
\hline Diarrhoea (DI) & $21.11(30.92)$ & $10.00(27.88)$ & 0.37 & 0.097 \\
\hline Financial (FI) & $78.89(29.66)$ & $65.55(33.31)$ & 0.41 & 0.009 \\
\hline
\end{tabular}

${ }^{\mathrm{a}}$ The higher score, the better the level of functioning

${ }^{\mathrm{b}}$ The higher the score, the worse the symptoms/problems

$P<0.05$ indicate significance 
Table 3 Demographic variables that effect quality of life ${ }^{a}$

\begin{tabular}{|c|c|c|c|c|c|c|c|c|c|c|}
\hline Variable & $\mathrm{GH}$ & $\mathrm{EF}$ & SF & FA & PA & DY & SL & AP & $\mathrm{CO}$ & $\mathrm{FI}$ \\
\hline \multicolumn{11}{|l|}{ Sex patient ${ }^{\mathrm{a}}$} \\
\hline Male & 0.038 & - & - & - & - & 0.046 & - & - & 0.030 & - \\
\hline \multicolumn{11}{|l|}{ Female } \\
\hline \multicolumn{11}{|l|}{ Age patient ${ }^{\mathrm{b}}$} \\
\hline$<44$ years old & 0.002 & - & - & - & - & - & - & - & - & 0.039 \\
\hline \multicolumn{11}{|l|}{ 45-54 years old } \\
\hline \multicolumn{11}{|l|}{$>55$ years old } \\
\hline \multicolumn{11}{|c|}{ Sex Family caregiver ${ }^{a}$} \\
\hline Male & - & - & - & - & - & - & - & - & - & - \\
\hline \multicolumn{11}{|l|}{ Female } \\
\hline \multicolumn{11}{|c|}{ Age Family Caregiver ${ }^{\mathrm{b}}$} \\
\hline$<44$ years old & - & - & - & - & - & 0.031 & - & - & - & - \\
\hline \multicolumn{11}{|l|}{ 45-54 years old } \\
\hline \multicolumn{11}{|l|}{$>55$ years old } \\
\hline \multicolumn{11}{|c|}{ Relationship with patient ${ }^{a}$} \\
\hline Spouse & - & - & - & - & - & - & - & - & - & - \\
\hline \multicolumn{11}{|l|}{ Non-spouse } \\
\hline \multicolumn{11}{|c|}{ Educational background of family caregiver ${ }^{\mathrm{b}}$} \\
\hline Elementary & - & - & - & - & - & - & - & - & - & - \\
\hline \multicolumn{11}{|l|}{ Junior high } \\
\hline \multicolumn{11}{|l|}{ Senior high } \\
\hline \multicolumn{11}{|l|}{ University } \\
\hline \multicolumn{11}{|c|}{ Experience in caregiving of family caregiver ${ }^{a}$} \\
\hline Yes & - & - & - & - & - & - & - & - & - & - \\
\hline No & & & & & & & & & & \\
\hline
\end{tabular}

scale that increased significantly after intervention, namely, global health status/QoL emotional and social functioning, as well as seven items on symptoms and single items, namely, fatigue, pain, dyspnea, insomnia, appetite loss, constipation, and financial issues.

A positive impact of intervention may result for several reasons. First, although McMillan and Weitzner (1998) suggested that the QoL of patients in terminal condition decreased mostly because of declining physical functioning, while their social and spiritual functioning were relatively high [17]. However, improved global health status/ QoL of patients in the present study may relate to emotional and social aspects that were facilitated during the intervention.

Second, the substantial increase of emotional and social functioning may be attributable to the intervention being developed mostly to enhance family caregiver involvement in palliative patient care [18]. Being together with close friends and family members is possibly the most important coping strategy for individuals facing impending death from cancer [19]. Providing constructive social support is also proven beneficial in reducing pain and lowering the number of depressive symptoms [20]. As participants in the current study were mostly immobile, family caregivers might become the crucial source for social support [21]. Provision of basic nursing care likely provides the opportunity for social conversation, which helps keep the patient engaged in family life. Emotional support also can be enhanced by involving the patients as active participants, as this involvement helps maintain the patients' dignity [22] and may enhance their personal sense of meaning through active engagement with palliative care providers [23, 24]. During the training, the nurse educators encouraged the family caregivers to involve the patients in their own care as much as possible.

Third, it is also possible that providing comfort measures, such as changing their positions and improving their hygiene, helps patients reduce focus on their physical discomfort and makes them more capable of staying socially and emotionally engaged [23]. From a palliative care perspective, most patients consider self-esteem and personal image to be important aspects. They want to stay physically clean and free from odors and bodily fluids, and want to have a normal appearance despite their being in the dying process [25].

In summary, most symptoms declined after an intervention given at a time when terminal patients tend to suffer a decline after hospital discharge. These improvements can be linked to the procedures provided in the intervention in the current study, such as showering, hair washing, feeding, assisting with toileting, physical repositioning, and oral hygiene. The latter is especially important in countering side effects of chemotherapy and radiation, such as dry mouth and decreased appetite [26].

A previous meta-analysis showed that interventions given to family caregivers of patients were divided into three types: psycho-education, skills training focused on coping and problem solving, and therapeutic counseling [27]. The study made no mention of skills training, which provides essential information for practical steps in handling routine daily living activities and personal care. One reason for this difference in protocols may be the meta-analysis' being limited to developed countries. Therefore, interventions conducted in the current research are considered more appropriate for the context of this pilot study, where many terminal cancer patients live out their life at home with their family as a palliative care provider, as is very common in Indonesia and much of Asia.

Family caregivers in Asian countries generally stay with the patient constantly. They are usually willing to provide any type of palliative care necessary. When the patient is 
staying at home and lacks ability to complete daily living activities, most family caregivers are not equipped to handle the challenges involved in assisting them [27]. Asian patients may also feel more comfortable receiving help from their own relatives. The current study used an educational package consisting of an instruction manual and informational video, along with demonstrations by nurse educators. The video was added to provide visual demonstration and make the manual's instructions easier to comprehend. Family caregivers can refer to the manual, which contains detailed explanation and pictures, when they need more information. Recent research on similar interventions using only manuals showed that health professionals used this as a complementary aid. Health professionals shared a variety of experiences in palliative care, and stated they were more comfortable using their own ways of providing information [28]. Therefore, an intervention's success may vary depending on the innovations health professionals produce.

\section{Strengths and limitations}

The current study measured the impact of family caregivers' skills training on patients' QoL, as there is a lack of evidence about specific nursing education interventions in this area [27], While limited by the small sample patient population in this pilot study $(n=30)$, the results are unique because there are few, if any, studies that directly address intervention protocols for the palliative care providers of terminal cancer patients from low- to medium-income countries [29]. However, a positive impact on QoL through palliative care has been demonstrated [30]. Our study was valuable because, to our knowledge, this was the first Indonesia-based study that attempted, by using an instructional module with a manual and supplemental video, to provide basic skills training for family caregivers of palliative cancer patients. The intervention also attempted to combine provision of an educational package with direct teaching sessions, and set up the follow-up at home to further encourage the family caregivers.

The main limitation of the present study was the absence of a control group. However, given the sensitive nature of the topic of death and dying, and the scarcity of research in the area of palliative care in Indonesia, a decision was made to delimit this initial study in order to assess acceptability of the outcome measures to this vulnerable population. Completion of the advance and follow-up questionnaires did not appear to cause any distress for the patients or their relatives. This opens the door for the next stage of investigation, which would be a randomized controlled trial of the intervention for palliative care with terminally ill cancer patients in Indonesia, or in other Southeast Asian countries with similar conditions.

\section{Conclusions}

The present study provides essential data, which will serve as a basis for subsequent investigations of the provision of palliative care in developing countries. It is recommended that a larger-scale study with the addition of a control group be conducted to further delineate the benefits of provision of BST to family members of cancer patients. Because the interventions and teaching aids herein were simple and affordable, it is also recommended that similar studies in urban, suburban, and rural areas be conducted to investigate differences in acceptance of the program. Given that financial aspects are one of Indonesians' main concerns, it is recommended that they also be taken into account in future research in an Indonesian setting. Finally, prior to any randomized control trial, it is suggested that psychometric adaptations are made to the EORTC QLQ C-30 to accommodate the economic and cultural sensitivities of terminally ill Indonesian patients, and to facilitate effective interpretation of the scores.

\section{Abbreviations \\ AP: Appetite loss; BST: Basic skills training; CF: Cognitive functioning; CO: Constipation; DI: Diarrhea; DY: Dyspnea; EF: Emotional functioning; EORTC QLQ C30: European Organization for Research and Treatment Cancer Quality of Life C30; FA: Fatigue; Fl: Financial hardship related to illness; NV: Nausea/vomiting; PA: Pain; PF: Physical functioning; PPS: Palliative performance scale; QoL: Quality of life; RF: Role functioning; SF: Social functioning; SL: Insomnia/sleep}

\section{Acknowledgements}

The authors thank patients and family caregivers for their study participation. The authors also thank the team of nurse educators for their support. Furthermore, the authors thank Dr Kathy Ahern, PhD for her advice in the manuscript.

\section{Funding}

The study was funded by Alumni Reference Group Research Seed Funding, Australia.

Availability of data and materials

The datasets analysed during the current study are available from the corresponding author on reasonable request

Authors' contributions

All authors have made substantial contributions to the conception and design of the study. MSK contributed to concept and designed of the study, produced training materials, carried out data collection, extracted the data, analysed the results, and drafted the manuscript. SS contributed to study design, produced training materials, participated in data analyses and drafted manuscript. CE contributed to study design, contributed to study materials, and critically revised the manuscript. All authors read and approved the final version.

Competing interests

The authors declare that they have no competing interests.

Consent for publication

Not applicable.

Ethics approval and consent to participate

An Ethical approval was granted from the Ethics Committee of the Faculty of Medicine, Universitas Gadjah Mada, Yogyakarta number KE/FK/528/EC.

Written informed consents were received from all participants. 
Received: 18 January 2016 Accepted: 23 December 2016

Published online: 17 January 2017

\section{References}

1. Dans A, Ng N, Varghese C, Tai ES, Firestone R, Bonita R. The rise of chronic non-communicable diseases in southeast Asia: Time for action. Health in Southeast Asia 4. 2011;377:680-9.

2. D'ambruoso L, Byass P, Qomariyah SN. 'Maybe it was her fate and maybe she ran out of the blood': Final caregivers' perspectives on access to care in obstetric emergencies in rural Indonesia. J Biosoc Sci. 2010;42(2):213-41.

3. Iskandarsyah A, Suardi D, Soemitro MP, de Klerk C, Passchier J, Sadarjoen SS. Psychosocial and cultural reasons for delay in seeking help and nonadherence to treatment in Indonesian women with breast cancer: A qualitative study. Health Psychology. 2013;21.

4. Howard SC, Pui CH, Ribeiro C. Components of cure: treatment of acute lymphoblastic leukimia in Indonesia and other low-income countries. Pediatric Blook Cancer. 2008:51:719-21.

5. Supit W, Mochtar CA, Sugiono M, Umbas R. Survival of patients with transitional cell carcinoma of the urinary bladder in Indonesia: A single institution review. Asian Pasific journal Cancer Prevention. 2011:12:549-53.

6. Sparrow R, Suryahadi A, Widyanti W. Social health insurance for the poor: targeting and impact of Indonesia's Askeskin programme. Social Science \& Medicine. 2013;96:264-71.

7. Halabi SF. Participation and right to health: Lessons from Indonesia. Health Hum Rights. 2009;11(1):49-59.

8. Ibrahim K, Haroen H, Pinxten L. Home-based care: a need assessment of people living with HIV infection in Bandung, Indonesia. J Assoc Nurses AIDS Care. 2011;22(3):229-37.

9. Mostert S, Gunawan S, Wolters E, Van de ven P, Sitaresmi MN, Van Dongen J, Veerman AJP, Mantik M, Kaspers G. Socio-economic status plays important role in childhood cancer treatment outcome in Indonesia. Asian Pac Cancer P. 2012;13:6491-96

10. Effendy C, Vissers K, Tejawinata S, Vernooij-Dassen M, Engels Y. Dealing with symptoms and issues of hospitalized patients with cancer in indonesia: the role of families, nurses, and physicians. Pain Practice: The Official Journal of World Institute of Pain. 2015;15(5):441-6.

11. Given B, Sherwood PR, Given CW. What knowledge and skills do caregivers need? Advanced Journal of Nursing. 2008;108(9):28-34.

12. Clemens K, Kumar S, Bruera E, Klaschik E, Jaspers B, De Lima L. Palliative care in developing countries: What are the important issues? Palliative Medicine. 2007;21:173-5.

13. Aaronson NK, Ahmedzai S, Bergman B, Bullinger M, Cull A, Duez NJ, Filiberti A, Flechtner H, Fleishman SB, de Haes JCJM, et al. The European Organization for Research and Treatment of Cancer QIQ-C30: A quality-of-life instrument for use in international clinical trials in oncology. J Natl Cancer Inst. 1993;85(365-376).

14. Fayers PM, Aaronson NK, Bjordal K, Groenvold M, Curran D, Bottomley A, on behalf of the EORTC Quality of Life Group. The EORTC QLQ-C30 Scoring Manual (3rd Edition). Published by: European Organisation for Research and Treatment of Cancer, Brussels; 2001

15. Perwitasari DY, Atthobari J, Dwiprahasto I, Hakimi M, Gelderblom H, Putter H, Nortier JWR, Guchelaar HJ, Kaptein AA. Translation and validation of EORTC QLQ C-30 into Indonesian version for cancer patients in Indonesia. Jpn J Clin Oncol. 2011:41(4):519-29.

16. Orwin RG. A fail-safe N for effect size in meta-analysis. J Educ Stat. 1983;8(2):157-9.

17. McMillan SC, Weitzner M. Quality of life in cancer patients. Cancer Pract. 1998;6(5):282-8

18. So WKW, Marsh G, Ling WM, Leung FY, Lo JCK, Yeung M, Li GKH. Anxiety, depression and quality of life among Chinese breast cancer patients during adjuvant therapy. Eur J Oncol Nurs. 2010;14:17-22.

19. Sand L, Olsson M, Strang P. Coping strategies in the presence of one's own impending death from cancer. J Pain Symptom Manage. 2009:37(1):13-22.

20. Hughes S, Jaremka LM, Alfano CM, Glaser R, Povoski SP, Lipari AM, Carson III WE, Malarkey WB, Kiecot-Glaser JK. Social support predicts inflammation, pain and depressive symptoms: Longitudinal relationships among breast cancer survivors. Psychoneuroendocrinology. 2014;42:38-44.

21. Cui J, Fang F, Shen F, Song L, Zhou L, Ma X, Zhao J. Quality of life in patients with advanced cancer at the end of life as measured by the McGill quality of life questionnaire: a survey in China. J Pain Symptom Management. 2014;48(5):893-902.

22. Östlund U, Brown H, Johnston B. Dignity conserving care at the end-of-life: a narrative review. Eur J Oncol Nurs. 2012;16:353-67.
23. Chan WCH. Can personal resources make a difference? an exploratory study of chinese cancer patients in palliative care. J Soc Work End Life Palliat Care. 2009;5(4):186-200

24. Cheng H, Sit JWH, Chan CWH, So WKW, Choi KC, Cheng KFK. Social support and quality of life among Chinese breast cancer survivors: Findings from a mixed methods study. Eur J Oncol Nurs. 2013;17:788-96.

25. Taveb MA, Al-Zamel E, Fareed MM, Abouellail HA. A "good death": Perspectives of Muslim patients and health care providers. Ann Saudi Medicine. 2010:30(3):215-21.

26. Wilberg P, Hjermstad MJ, Ottesen S, Herlofson BB. Oral health is an important issue in end-of-life cancer care. Support Care Cancer. 2012;20: 3115-22.

27. Northouse LL, Katapodi MC, Song L, Zhang L, Mood DW. Interventions with family caregivers of cancer patients: Meta- Analysis of Randomized Trials. CA Cancer J Clin. 2011:60(5):317-39.

28. Holm M, Carlander I, Fürst C-J, Wengström Y, Årestedt K, Öhlen J, Henriksson A. Delivering and participating in a psycho-educational intervention for family caregivers during palliative home care: a qualitative study from the perspectives of health professionals and family caregivers. BMC Palliative Care. 2015;14(1):1-10.

29. Harding R, List S, Epiphaniou E, Jones H. How can informal caregivers in cancer and palliative care be supported? An updated systematic literature review of interventions and their effectiveness. Palliative Medicine. 2012;26:7-22.

30. Strupeit S, Bup A, Dassen T. Effectiveness of nurse-delivered patients education interventions on quality of life in outpatiens: a systematic review. Appl Nurs Res. 2013;26:232-8.

\section{Submit your next manuscript to BioMed Central and we will help you at every step:}

- We accept pre-submission inquiries

- Our selector tool helps you to find the most relevant journal

- We provide round the clock customer support

- Convenient online submission

- Thorough peer review

- Inclusion in PubMed and all major indexing services

- Maximum visibility for your research

Submit your manuscript at www.biomedcentral.com/submit
Biomed Central 